\title{
First-Principles Calculation of Transport Coefficients
}

\author{
Dario Alfè \\ Geological Sciences Department, University College London, Gower Street, London WClE 6BT, United Kingdom \\ Michael J. Gillan \\ Physics Department, Keele University, Keele, Staffordshire ST5 5BG, United Kingdom
}

(Received 7 May 1998)

\begin{abstract}
We demonstrate the practical feasibility of calculating transport coefficients such as the viscosity of liquids completely from first principles using the Green-Kubo relations. Results presented for liquid aluminum are shown to have a statistical error of only ca. 5\%. The importance of such calculations is illustrated by results for a liquid iron-sulfur alloy under Earth's core conditions, which indicate that the viscosity of the liquid outer core is no more than an order of magnitude higher than that of typical liquid metals under ambient conditions. [S0031-9007(98)07869-7]

PACS numbers: $66.20 .+\mathrm{d}, 71.15 . P d$
\end{abstract}

Since the pioneering work of Car and Parrinello [1] first-principles molecular dynamics (FPMD) has become a widely used technique for investigating condensed matter. For liquids and solids in thermal equilibrium many quantities of interest are readily calculated, including thermodynamic functions, structure factors, radial distribution functions, diffusion coefficients, bond lifetimes, etc. But transport coefficients such as viscosity and thermal conductivity are computationally more demanding, and have been little studied by FPMD. We demonstrate here the feasibility of calculating such quantities with useful accuracy, by presenting FPMD results for the viscosity of liquid aluminum near the triple point. We then illustrate the potential importance of this type of calculation by showing how FPMD calculations can be used to calculate the viscosity of liquid iron in the earth's outer core. This is one of the key quantities in the theory of the earth's deep interior, but also one of the most uncertain.

There are basically two ways of calculating transport coefficients by simulation. The first is to subject the simulated system to an explicit external perturbation (e.g., a shear flow or a temperature gradient) and calculate the steady-state response. Alternatively, one can apply the Green-Kubo (GK) relations, i.e., the relations between transport coefficients and correlation functions involving fluxes of conserved quantities [2]. The shear viscosity $\eta$, for example, is given by

$$
\eta=\frac{V}{k_{B} T} \int_{0}^{\infty} d t\left\langle P_{x y}(t) P_{x y}(0)\right\rangle,
$$

where $\langle\cdot\rangle$ denotes the thermal average, $V$ is the volume of the system, $T$ is the temperature, $k_{B}$ is the Boltzmann factor, and $P_{x y}$ is the off-diagonal component of the stress tensor $P_{\alpha \beta}$ ( $\alpha$ and $\beta$ are Cartesian components). The relative merits of the two approaches have been much discussed, but the GK method has the virtues of simplicity and ease of application, and will be used here.

For a simulation having a given duration, single particle properties, such as the diffusion coefficient, can be calculated more accurately than collective properties, such as the viscosity. In the former case, the statistical average can be done over time and over the particles, while, in the latter, one loses the possibility of averaging over the particles. To obtain the same statistical accuracy, collective properties need much longer runs than single particle properties by a factor proportional to the size of the system.

The first-principles calculations presented here are based on density functional theory, pseudopotentials, and plane-wave basis sets. The electron-ion interaction is described by ultrasoft Vanderbilt pseudopotentials [3]. Nonlinear core corrections [4] are included throughout this work. The calculations have been performed using VASP (Vienna ab initio simulation package) [5]. In VASP the electronic ground state is calculated exactly (within a self-consistency threshold) at each MD step, using an efficient iterative matrix diagonalization scheme and a Pulay mixer [6]. Within this approach to FPMD it is important to provide a good starting electronic charge density at each time step, so as to reduce the number of iterations to achieve self-consistency. We have used the following charge extrapolation scheme: at the beginning of each time step, the electronic charge density is extrapolated using the atomic charge density and a quadratic extrapolation on the difference; i.e., the charge is written as $\rho(t)=\rho_{\text {at }}(t)+\delta \rho(t)$, where $\rho(t)$ is the self-consistent charge density at time $t$, and $\rho_{\text {at }}(t)$ is the sum of the atomic charges. At time $t+d t$ the charge is written as the sum of the atomic charges, which can be calculated exactly and cheaply, and a quadratic extrapolation on $\delta \rho$. This scheme provides a much better starting charge than the standard quadratic extrapolation of the whole charge [7]. The electronic levels are occupied according to Fermi statistics corresponding to the temperature of the simulation. This prescription also avoids problems with level crossing during the selfconsistent cycles. Forces and stress tensor are calculated using the Hellmann-Feynman theorem. The temperature is controlled using a Nosé thermostat [8]. 
Our calculation of the viscosity of liquid aluminum [9] was performed using the local density approximation (LDA) [10] for the exchange-correlation energy and a plane-wave cutoff of $130 \mathrm{eV}$. The temperature was $1000 \mathrm{~K}(70 \mathrm{~K}$ above the melting point) and the pressure was close to zero. We used a cell containing 64 atoms with periodic boundary conditions and $\Gamma$-point sampling only. (We note that $\Gamma$-point sampling is expected to be a good approximation for calculating the structure and dynamics of liquid metals, even though this would not be satisfactory for calculating such quantities as the electrical conductivity [11].) To integrate Newton's equation of motion for the ions, we used the Verlet [12] algorithm with a time step of $3 \mathrm{fs}$, and the convergence threshold on the total energy was $1.5 \times 10^{-8} \mathrm{eV} /$ atom; with these prescriptions the drift in the total energy was $\approx 0.2 \mathrm{meV} /$ atom per ps. The length of the simulation was $80 \mathrm{ps}$. The simulation was split into three parts, of lengths $\approx 40 \mathrm{ps}, \approx 25 \mathrm{ps}$, and $\approx 15 \mathrm{ps}$, and at the beginning of each part the Nosé thermostat was reinitialized, so as to avoid possible problems with the accumulation of the drift in the total energy. The cell volume was adjusted to give zero pressure; this resulted in a density of $\rho=2470 \mathrm{~kg} \mathrm{~m}^{-3}$, which is about 5\% larger than the experimental one [13]. This discrepancy is probably due mostly to the LDA.

There are five independent components of the traceless stress tensor: $P_{x y}, P_{y z}, P_{z x}, \frac{1}{2}\left(P_{x x}-P_{y y}\right)$, and $\frac{1}{2}\left(P_{y y}-\right.$ $\left.P_{z z}\right)$, the last two being equivalent to the first three by rotational invariance, so one can construct five independent stress autocorrelation functions (SACF). These are calculated by averaging $P_{\alpha \beta}\left(t+t_{0}\right) P_{\alpha \beta}\left(t_{0}\right)$ over time origins $t_{0}$. Since reinitialization of the thermostat causes small discontinuities, this averaging is restricted so that $t_{0}$ and $t+t_{0}$ never span a discontinuity.

In Fig. 1, we display the average $\phi(t)$ of the five SACF's divided by its value at $t=0$ for the aluminum system. Since the traceless part of the stress tensor has zero average, $\phi(t)$ goes to zero as $t \rightarrow \infty$. The statistical error on $\phi(t)$ for all values of $t$ is $\approx 1.5 \%$ of the value at $t=0$, and after $0.3-0.4 \mathrm{ps}$ the magnitude of $\phi(t)$ falls below that error.

In Fig. 2, we display the integral $\int_{0}^{t} d t^{\prime} \phi\left(t^{\prime}\right)$ of $\phi(t)$ as a function of time. The limit value of the integral for $t \rightarrow \infty$ is the shear viscosity. The error that one makes in evaluating that integral grows with time, since one integrates the noise together with $\phi(t)$. We estimated the error in the integral as a function of time using the scatter of the SACF's calculated by splitting the simulation into many short intervals. We combined this estimate with an analytic expression for the error, and the resulting error estimate is displayed in Fig. 2. From the point where $\phi(t)$ falls below the noise, one integrates only the latter, so one gains nothing by evaluating the integral beyond that point. If we assume that $\phi(t)$ is zero above $t \approx 0.4 \mathrm{ps}$, we obtain the value $\eta=2.2 \pm 0.1 \mathrm{mPas}$, which should be compared with the experimental value of

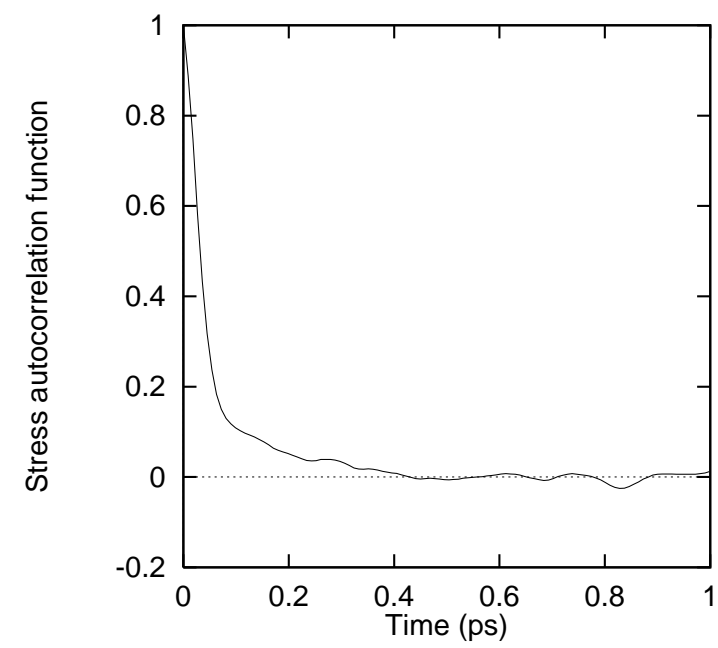

FIG. 1. Average over the five independent components of the autocorrelation function of the traceless stress tensor $\phi(t)$ calculated for liquid $\mathrm{Al}$ at $1000 \mathrm{~K}$ at the density of $2470 \mathrm{~kg} \mathrm{~m}^{-3}$. Values of $\phi(t)$ are normalized by dividing by $\phi(0)$.

$1.25 \mathrm{mPa}$ [ [14]. The fact that the statistical errors have been reduced to $\approx 5 \%$ demonstrates that the viscosity of liquids can be calculated completely from first principles with quite high precision. Our calculated value of the viscosity differs significantly from the experimental one. In order to find a possible reason for this discrepancy, we have simulated for $20 \mathrm{ps}$ the aluminum system at the experimental density, $\rho=2350 \mathrm{~kg} \mathrm{~m}^{-3}$ (which gives a calculated negative pressure of $2 \mathrm{GPa}$ ). The results for the viscosity of this simulation are also reported in Fig. 2. We obtain $\eta=1.4 \pm 0.15 \mathrm{mPas}$, which is in excellent agreement with the experimental data.

A method which has sometimes been used in the past to obtain an approximate estimate of the shear viscosity exploits its connection with the self-diffusion coefficient $D$ via the Stokes-Einstein relation: $D \eta=k_{B} T / 2 \pi a$, where $a$ is an effective atomic diameter. This relation is exact for the Brownian motion of a macroscopic particle of diameter $a$ in a liquid of viscosity $\eta$, but it is only approximate when applied to atoms. However, if $a$ is chosen to be the radius $r_{1}$ of the first peak in the radial distribution function, the relation usually predicts $\eta$ to within $40 \%$. We have calculated $D$ from our simulated liquid aluminum in the standard way from the long time slope of the mean-square displacement $\left\langle\left|\mathbf{r}_{i}\left(t_{0}+t\right)-\mathbf{r}_{i}\left(t_{0}\right)\right|^{2}\right\rangle \rightarrow 6 D t$, as $t \rightarrow \infty$, where $\mathbf{r}_{i}$ is the position of atom $i$. The resulting value $D \approx$ $5.2 \times 10^{-9} \mathrm{~m}^{2} \mathrm{~s}^{-1}$, combined with the value $a=r_{1}=$ $2.65 \AA$, gives $\eta \approx 1.6 \mathrm{mPa}$, which agrees quite well with the Green-Kubo value. For the system at the experimental density, we calculate $D \approx 6.8 \times 10^{-9} \mathrm{~m}^{2} \mathrm{~s}^{-1}$, which is consistent with the value of the viscosity.

Although the statistical errors on $\eta$ are small, one might ask whether a 64-atom system is big enough to calculate $\eta$ accurately. The effect of system size on calculated 
viscosities has been extensively studied for models such as the hard-sphere and Lennard-Jones liquids—close packed systems which are similar to liquid aluminum. For example, Schoen and Hoheisel [15] examined Green-Kubo calculations of the Lennard-Jones liquid for system sizes from 32 to 2048 atoms. They showed that even 32 atoms give meaningful values for $\eta$ and that for 64 atoms the error on $\eta$ at the triple point is $\approx 10 \%$. We note that one indicator of system size effects should be the difference between $\eta$ values calculated with the stress components $P_{x y}$, etc., and those calculated from $\frac{1}{2}\left(P_{y y}-P_{z z}\right)$, etc., since cubic periodicity breaks the spherical equivalence of the two types of SACF. We searched for this difference in our calculations, but it is not large enough to be clearly visible above the statistical noise.

Liquid iron under Earth's core conditions provides a good example of the importance of being able to calculate transport coefficients by FPMD. The earth's liquid outer core consists mainly of molten iron, with light impurities, of which sulfur is a likely candidate [16]. The shear viscosity of this liquid is a crucial factor in understanding convective circulation in the core, which is intimately linked both to heat transport and to the generation of the earth's magnetic field. Nevertheless it is one of the most uncertain quantities in geophysics, with estimates from different experimental and theoretical methods spanning no less than 12 orders of magnitude [17]. A firmly based calculation of the viscosity is therefore important. In our recent FPMD calculations for liquid $\mathrm{Fe}[18,19]$ and liquid Fe-S [7] under Earth's core conditions, we used the StokesEinstein relation to obtain the estimate $\eta=13 \mathrm{mPa}$ s. But the use of this relation under such extreme conditions can clearly be challenged.

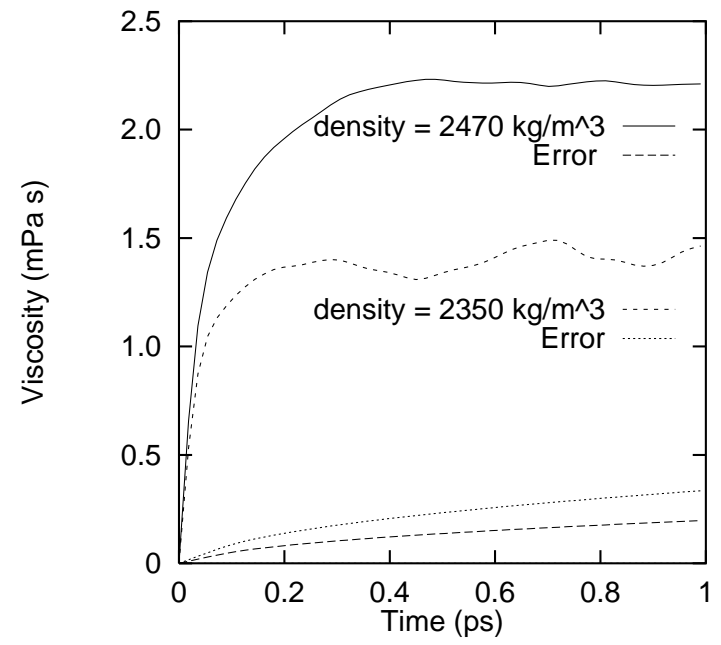

FIG. 2. Viscosity integral of the average stress autocorrelation function and its statistical error as a function of time for liquid $\mathrm{Al}$ at $1000 \mathrm{~K}$. Results are shown for the calculated zero pressure density $\rho=2470 \mathrm{~kg} \mathrm{~m}^{-1}$ and the experimental density $\rho=2350 \mathrm{~kg} \mathrm{~m}^{-1}$.
We present here the results that we have obtained from a direct calculation of the viscosity of an Fe-S liquid alloy under Earth's core conditions. The calculations were performed at a thermodynamic state corresponding to the boundary between the solid inner core and the liquid outer core. In fact, the temperature at this boundary is uncertain, with estimates ranging from 4000 to $8000 \mathrm{~K}$, while the density is quite accurately known to be about $12000 \mathrm{~kg} \mathrm{~m}^{-3}$ [20]. The temperature in our simulation was set to $6000 \mathrm{~K}$ and the density was $12330 \mathrm{~kg} \mathrm{~m}^{-3}$. The Fe-S mixture was taken to have a sulfur mole fraction of 0.1875 , in line with the maximum estimates for sulfur abundance in the core [21]. The simulated system contained 64 atoms ( $52 \mathrm{Fe}$ and $12 \mathrm{~S}$ ) in the repeated cell, with $\Gamma$-point sampling only. We have used the generalized gradient approximation [22] for the exchange-correlation energy, a plane-wave cutoff of $350 \mathrm{eV}$, a time step of $1 \mathrm{fs}$, and a self-consistency threshold of $1.5 \times 10^{-7} \mathrm{eV} /$ atom. The total length of the simulation was $10 \mathrm{ps}$ and the drift on the total energy was $\approx 8 \mathrm{meV} /$ atom per ps. A complete description of the results of this simulation is reported elsewhere [7].

In Fig. 3, we display the average SACF $\phi(t)$ for this system. This is noisier than for the aluminum system, the statistical error at any time being $\approx 4 \%$ of $\phi(t=0)$, but it is still possible to produce a useful estimate for the viscosity. The $\phi(t)$ has gone to zero after $t \approx 0.2 \mathrm{ps}$ (Fig. 3), and the time integral of $\phi(t)$ up to this point gives $\eta=9 \pm 2 \mathrm{mPa}$ s (Fig. 4), which confirms the approximate correctness of the value estimated using the Stokes-Einstein relation. The error has been estimated as in the aluminum case. This value of $\eta$ is about 10 times larger than the viscosity of typical liquid metals at ambient pressure. This result is important since it provides concrete support for the approximation commonly made in

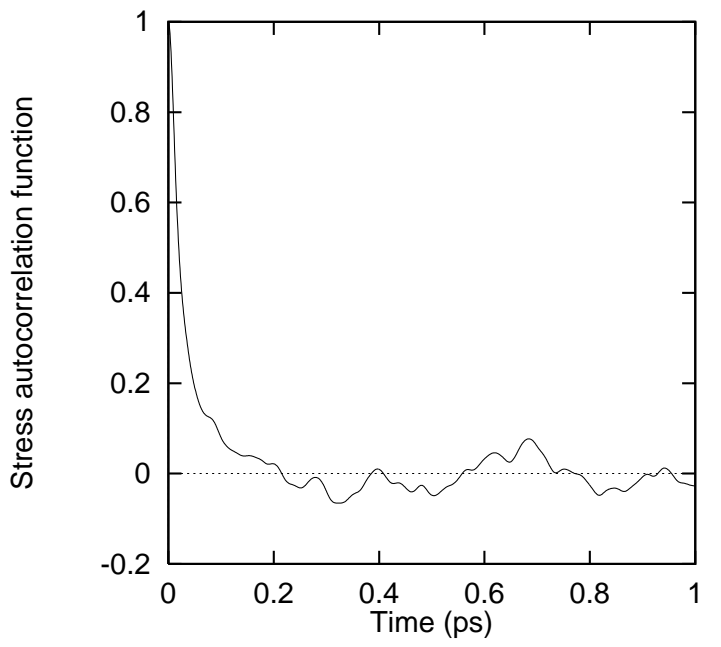

FIG. 3. Average over the five independent components of the autocorrelation function of the traceless stress tensor $\phi(t)$ calculated for liquid Fe-S under Earth's core conditions. Values of $\phi(t)$ are normalized by dividing by $\phi(0)$. 


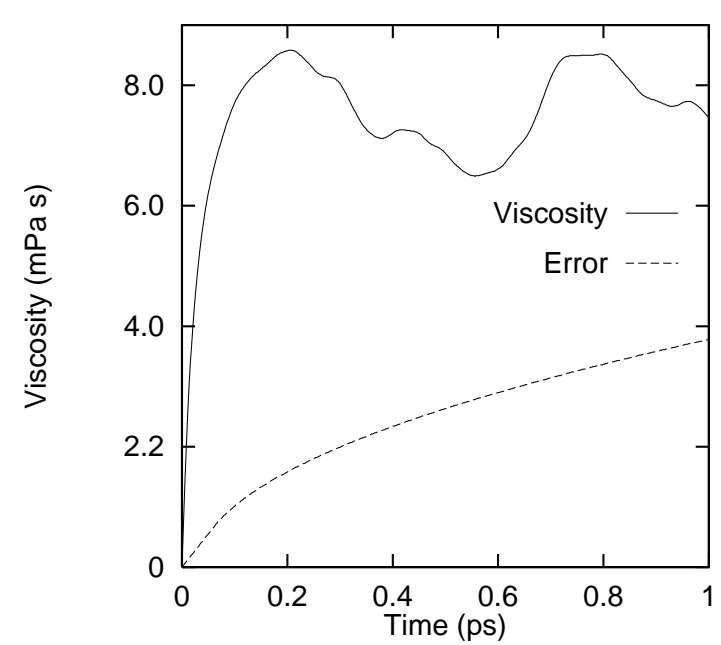

FIG. 4. Viscosity integral of the average stress autocorrelation function and its statistical error as a function of time for liquid Fe-S under Earth's core conditions.

magnetohydrodynamic models that the outer core is an inviscid fluid $[23,24]$ undergoing small-circulation turbulent convection [25] rather than large-scale global circulation.

We have chosen the case of liquid Fe-S to illustrate the geophysical importance of first-principles calculations of viscosity, but there are many other areas where calculated viscosities would be valuable. The viscosity of the highly compressed hydrogen/helium mixtures in the deep interior of the giant planets is a case in point. There have been recent attempts to deduce this viscosity from measured gravitational anomalies for Jupiter, and input from firstprinciples calculations obtained using the methods we have described would be very useful. Insight into the percolation of molten minerals in the earth and the other terrestrial planets could also be gained by first-principles methods. In a completely different context, the viscous flow of materials is crucial in a wide variety of industrial processes.

Although we have focused here on the shear viscosity, first-principles calculations should also be feasible for other transport coefficients such as the bulk viscosity, the thermal conductivity, and chemical interdiffusion coefficients, all of which contribute to the attenuation of sound in fluids.

In conclusion, we have demonstrated the feasibility of calculating transport coefficients such as viscosity from first principles, and we have presented results for the viscosity of liquid aluminum which agree well with experiment. We illustrated the importance of such calculations by presenting results for the viscosity of liquid $\mathrm{Fe}-\mathrm{S}$ under Earth's core conditions. These results support recent approximate estimates which indicate that the viscosity of the earth's outer core is in the region of $10 \mathrm{mPa}$. This is far lower than the values sometimes inferred from seismic and other measurements, and has important implications for the understanding of circulation in the core. Finally, we have pointed out that the techniques employed should also be applicable to other transport coefficients.

We acknowledge the support of NERC under Grant No. GST/O2/1454 and computer time allocated by the High Performance Computing Initiative.

[1] R. Car and M. Parrinello, Phys. Rev. Lett. 55, 2471 (1985).

[2] M.P. Allen and D. J. Tildesley, Computer Simulation of Liquids (Clarendon, Oxford, 1987).

[3] D. Vanderbilt, Phys. Rev. B 41, 7892 (1990).

[4] S. G. Louie, S. Froyen, and M. L. Cohen, Phys. Rev. B 26, 1738 (1982).

[5] G. Kresse and J. Furthmüller, Phys. Rev. B 54, 11169 (1996); G. Kresse and J. Furthmüller, Comput. Mater. Sci. 6, 15 (1996); a discussion of the ultrasoft pseudopotentials used in the VASP code is given in G. Kresse and J. Hafner, J. Phys. Condens. Matter 6, 8245 (1994).

[6] P. Pulay, Chem. Phys. Lett. 73, 393 (1980).

[7] D. Alfè and M. J. Gillan, Phys. Rev. B 58, 8248 (1998).

[8] S. Nosé, Mol. Phys. 52, 255 (1984); J. Chem. Phys. 81, 511 (1984).

[9] Our simulations of liquid aluminum build on earlier work reported in G.A. de Wijs, G. Kresse, and M. J. Gillan, Phys. Rev. B 57, 8223 (1998).

[10] D. M. Ceperley and B. J. Alder, Phys. Rev. Lett. 45, 566 (1980); we use the parametrization by J.P. Perdew and A. Zunger, Phys. Rev. B 23, 5048 (1981).

[11] P. L. Silvestrelli, A. Alavi, and M. Parrinello, Phys. Rev. B 55, 15515 (1997).

[12] L. Verlet, Phys. Rev. 159, 98 (1967).

[13] CRC Handbook of Chemistry and Physics, edited by D. R. Lide (CRC Press, New York, 1997), 78th ed.

[14] M. Shimoji and T. Itami, Atomic Transport in Liquid Metals (Trans Tech Publications, Aedermannsdorf, 1986).

[15] M. Schoen and C. Hoheisel, Mol. Phys. 56, 653 (1985).

[16] T. J. Usselmann, Am. J. Sci. 275, 278 (1975); Am. J. Sci. 275, 291 (1975); D. M. Sherman, Earth Planet. Sci. Lett. 132, 87 (1995).

[17] R. A. Secco, in Mineral Physics and Crystallography: A Handbook of Physical Constants, edited by T. J. Ahrens (American Geophysical Union, Washington, DC, 1995).

[18] L. Vočadlo, G. A. de Wijs, G. Kresse, M. J. Gillan, and G. D. Price, Faraday Discuss. 106, 205 (1997).

[19] G. A. de Wijs, G. Kresse, L. Vočadlo, D. Dobson, D. Alfè, M. J. Gillan, and G. D. Price, Nature (London) 392, 805 (1998).

[20] A. M. Dziewonski and D. L. Anderson, Phys. Earth Planet. Inter. 25, 297 (1981).

[21] T. J. Ahrens, J. Geophys. Res. 89, 6041 (1984).

[22] Y. Wang and J. Perdew, Phys. Rev. B 44, 13298 (1991).

[23] G. A. Glatzmaier and P. H. Roberts, Nature (London) 377, 203 (1995).

[24] R. T. Merril and P.L. McFadden, J. Geophys. Res. 100, 317 (1995).

[25] P. Melchior, The Physics of the Earth's Core (Pergamon Press, Oxford, 1986). 